\title{
Adição de grafite na conformação de substratos cerâmicos porosos processados por rolos a frio
}

\section{(Addition of graphite in the conformation of ceramic porous substrates processed by cold roll pressing)}

\author{
L. Koshimizu', M. R. Morelli ${ }^{2}$ \\ ${ }^{1} P P G-C E M,{ }^{2}$ Departamento de Engenharia de Materiais - DEMa \\ Universidade Federal de S. Carlos, Rod. Washington Luís, km 235, S. Carlos, SP 13565-905 \\ laiskoshimizu@yahoo.com.br
}

\begin{abstract}
Resumo
O desenvolvimento de substratos cerâmicos porosos de fina espessura $(<1 \mathrm{~mm})$ apresenta grande interesse tecnológico, podendo ter aplicações em diversas áreas, dentre as quais se destacam as eletrônicas e catalíticas. O processo viscoplástico tem se destacado como método de conformação cerâmica por minimizar a quantidade de líquido adicionado e a aglomeração de partículas durante o processamento, a partir de intenso cisalhamento da massa. Este processo permite a conformação de corpos cerâmicos de modo mais simples e sem aditivos tóxicos como na colagem de fita, método normalmente utilizado. Neste trabalho os substratos foram conformados por rolos a frio com espessura entre 400 e $700 \mu \mathrm{m}$, e velocidade dos rolos laminadores de $15 \mathrm{rpm}$. Estes foram secos antes de ser submetidos a queima a $1600{ }^{\circ} \mathrm{C}$ por $1 \mathrm{~h}$. Como agente porogênico foi estudada a grafite em pó que foi adicionada a massa plástica de alumina em duas concentrações diferentes, $9 \%$ e $18 \%$ em peso, a qual resultou numa porosidade aparente de $21 \%$ e $52 \%$ respectivamente.
\end{abstract}

Palavras-chave: conformação plástica, substratos cerâmico poroso, grafite.

\begin{abstract}
The development of porous ceramic substrates with thickness less than $1 \mathrm{~mm}$ presents a great technology interesting for electroelectronic or catalytic applications. Among all the techniques of ceramic conformation, the viscoplastic processing shows great advantages decreasing the amount of liquids and the particles agglomeration from an intensive shearing of the ceramic paste. This process allows the conformation of ceramic samples in a simple way and without toxic additives, the opposite of tape casting, which is the method often applied. The substrates were conformed by cold roll pressing in a thickness between 400 and $700 \mu \mathrm{m}$, and with the rotation of rollers of $15 \mathrm{rpm}$. Theses were dried before the firing at $1600^{\circ} \mathrm{C}$ for $1 \mathrm{~h}$. As a pore-forming agent it was studied the graphite powder which was added to the alumina plastic paste in two different concentrations, 9 and 18 wt\%, which results in an apparent porosity of 21 and $52 \%$, respectively.
\end{abstract}

Keywords: plastic conformation, porous ceramic substrate, graphite.

\section{INTRODUÇÃO}

As cerâmicas porosas apresentam um grande potencial para muitas aplicações onde os materiais metálicos ou poliméricos tornam-se inapropriados, como em isolamento térmico, filtros, membranas ou biocerâmicas [1-3]. As membranas cerâmicas exibem inúmeras vantagens tais como elevada eficiência de separação, excelente estabilidade térmica, estabilidade química e facilidade na limpeza quando comparada às membranas orgânicas ou metálicas. Por isso as membranas cerâmicas têm sido muito estudadas nas últimas duas décadas. Hoje, as áreas mais relevantes de membranas cerâmicas estão focadas principalmente em melhorar o desempenho e reduzir os custos de fabricação [4]. Para um bom desempenho é necessária uma boa distribuição de tamanho de poros para maximizar a função desejada, sendo que para diferentes aplicações, distintos níveis de porosidade são requeridos [5]. As várias aplicações dessas estruturas porosas exigem uma diversidade de necessidades combinando a quantidade de poros com suas características, tais como tamanho, formato, localização, grau de interconexão e orientação [6]. Cerâmicas porosas podem ser produzidas com a adição de agentes porogênicos como serragem, amido, carbono ou partículas orgânicas as matérias primas [7, 8]. Para uma aplicação bem sucedida desses materiais avançados o controle da microestrutura e a adequação das propriedades físicas necessárias, tais como resistência mecânica, a choque térmico e tenacidade a fratura, são de extrema importância. Para alcançar as melhores condições de sinterização são necessárias propriedades adequadas do material, tolerância dimensional, empacotamento de partículas homogêneo 
Tabela I - Composições das massas plásticas (\% massa) utilizada para obtenção de substratos porosos por conformação por rolos a frio.

[Table I - Plastic paste compositions (\% weight) to obtain ceramic porous substrates conformed by cold roll pressing.]

\begin{tabular}{cccccc}
\hline Material & Cerâmica & Lubrificante & Plastificante & Solvente & $\begin{array}{c}\text { Agente } \\
\text { Porogênico }\end{array}$ \\
\hline Composição 1 & $66 \%$ & $10 \%$ & $1 \%$ & $14 \%$ & $9 \%$ \\
Composição 2 & $60 \%$ & $9 \%$ & $1 \%$ & $12 \%$ & $18 \%$ \\
\hline
\end{tabular}

e distribuição uniforme de aditivos. Novos métodos de processamento têm sido constantemente desenvolvidos a fim de cumprir esses requisitos, assim como um processo com custo menor, ambientalmente correto e reprodutível [9].

Dentre as diversas técnicas de conformação cerâmica, o processo viscoplástico (conformação plástica) tem se destacado, uma vez que pode minimizar a aglomeração de partículas durante o processamento, usualmente a partir de intenso cisalhamento dos componentes. Normalmente as massas plásticas são preparadas por meio da mistura dos pós, aditivos e água, o que, na maioria das vezes ocasiona a aglomeração das partículas. No entanto, a correta seleção de aditivos orgânicos e um intenso cisalhamento podem promover a obtenção de uma massa viscoplástica uniforme que resultará em uma microestrutura mais homogênea após a conformação e queima. Este processo, conhecido como VPP (Viscous Plastic Processing), permite a conformação de corpos cerâmicos com excelentes valores de resistência mecânica, quando comparados aos obtidos em técnicas tradicionais, como o processo de prensagem e colagem [10].

Para o processo de conformação plástica existem vários fatores que afetam o rendimento do processo e a qualidade do produto final, entre eles destacam-se a composição e preparação da massa cerâmica, a plasticidade da massa, o tipo de equipamento e a qualidade do vácuo [11]. Esta é uma rota alternativa que pode ser aplicada à conformação de substratos cerâmicos, por meio da laminação da massa viscoplástica por rolos a frio. Por ser relativamente simples, porém recente, e apesar de ser atrativa economicamente para a aplicação em processos industriais, não há muitos registros em literatura sobre as principais variáveis envolvidas neste tipo de processamento, nem das características físicas e microestruturais dos substratos obtidos [12].

O desenvolvimento de substratos cerâmicos porosos de fina espessura $(<1000 \mu \mathrm{m})$ apresenta grande interesse tecnológico para o uso em substratos assimétricos em estruturas multicamadas para aplicações eletrônicas, catalíticas ou filtrantes [13]. A alumina é frequentemente escolhida como matéria prima para membrana cerâmica devido a sua elevada resistência e estabilidade térmica e química [14]. Neste âmbito, o objetivo deste trabalho foi avaliar a possibilidade de conformar substratos cerâmicos porosos por meio do processamento viscoplástico, para tanto, foi estudado como formador de poros a grafite em pó em uma matriz de alumina.

\section{MATERIAIS E MÉTODOS}

Duas composições de massas plásticas de alumina foram preparadas para a conformação de substratos de alumina porosos, variando a proporção do agente porogênico, $9 \%$ e 18\%. Dentre as matérias-primas estão a alumina (A1000, Almatis, EUA), que apresenta as seguintes características: D50 0,50 $\mu \mathrm{m}$, área superficial específica $8,2 \mathrm{~m}^{2} \mathrm{~g}^{-1}$, densidade $3,99 \mathrm{gm}^{-3}$ e $99,8 \%$ de pureza, e o pó de grafite (Micrograf, Nacional de Grafite Ltda.). As concentrações dos aditivos orgânicos utilizados nas formulações são apresentadas na Tabela I.

As massas plásticas de alumina foram preparadas em um misturador tipo sigma (Lieme - Modelo Liemita) e as etapas de preparação da massa plástica de alumina e da conformação do substrato cerâmico estão esquematizados no fluxograma da Fig. 1.

A massa obtida foi passada entre os rolos laminadores com velocidade de $15 \mathrm{rpm}$, sucessivas vezes e com distâncias cada vez menor entre os rolos laminadores, até atingir a espessura desejada do filme, variando entre 400 e $700 \mu \mathrm{m}$.

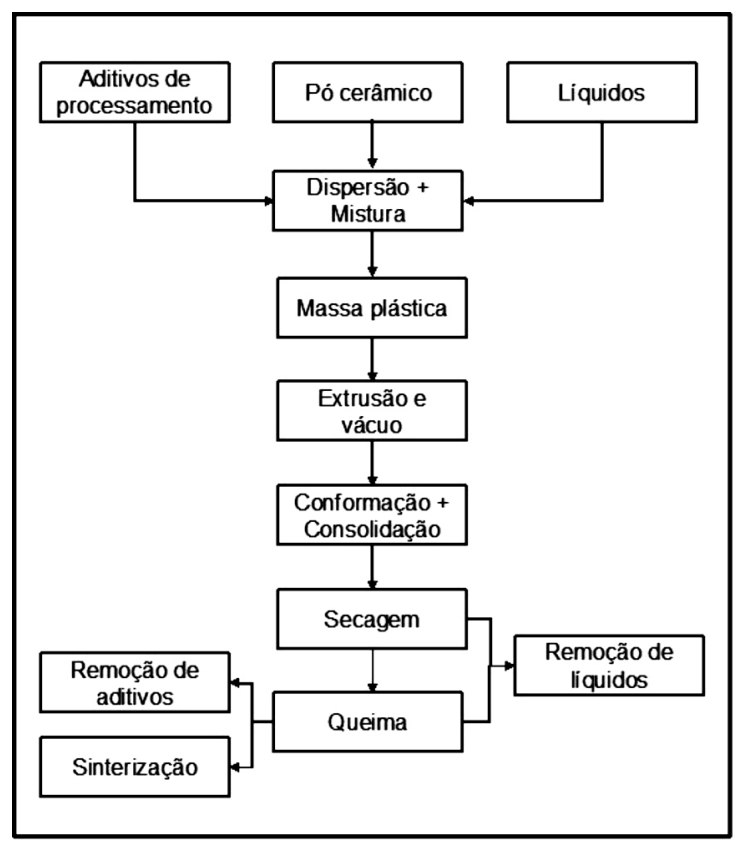

Figura 1: Fluxograma representativo de processamento por conformação viscoplástica de massas plásticas cerâmicas [7].

[Figure 1: Flow chart illustrative of viscoplastic conformation processing of ceramic plastic paste [7].] 
Com o intuito de verificar a temperatura na qual ocorre a eliminação dos compostos voláteis (aditivos) para o estabelecimento da curva de queima dos substratos de alumina foram feitas a análise térmica diferencial e a análise termogravimétrica; a atmosfera utilizada foi ar, mistura de oxigênio e nitrogênio, e a taxa de aquecimento foi de $5{ }^{\circ} \mathrm{C} /$ min até $900{ }^{\circ} \mathrm{C}$ (TA Instr. HiRes TGA 2950). Os substratos (seção quadrada $5 \mathrm{~cm} \times 5 \mathrm{~cm}$ ) foram armazenados por pelo menos $24 \mathrm{~h}$ a temperatura ambiente para a secagem. Posteriormente, foram queimados até $1600{ }^{\circ} \mathrm{C}$ durante $1 \mathrm{~h}$, com um patamar de $20 \mathrm{~min}$ a $700{ }^{\circ} \mathrm{C}$, e em seguida, resfriados até a temperatura ambiente.

\section{Caracterização dos substratos porosos}

A caracterização física dos substratos porosos conformados por rolos a frio foi feita por meio de medidas de densidade aparente (Eq. A) e porosidade aparente (Eq. B), pelo método de imersão em água baseado no princípio de Archimedes, de acordo com as equações apresentadas a seguir (Balança Ohaus Precision Plus).

A densidade aparente (DA) é calculada a partir da Eq. A.

$$
\mathrm{DA}=\frac{\mathrm{M}_{\mathrm{S}}}{\mathrm{M}_{\mathrm{u}}-\mathrm{M}_{\mathrm{i}}} \rho\left(\mathrm{g} / \mathrm{cm}^{3}\right)
$$

A porosidade aparente (PA) é obtida por meio da Eq. B.

$$
\% \mathrm{PA}=\frac{\mathrm{M}_{\mathrm{U}}-\mathrm{M}_{\mathrm{S}}}{\mathrm{M}_{\mathrm{U}}-\mathrm{M}_{\mathrm{I}}} \times 100
$$

nas quais $M_{s}$ é a massa da amostra seca, $M_{u}$ é a massa da amostra úmida, $\mathrm{M}_{\mathrm{i}}$ é a massa da amostra imersa em água, e $\rho$ é a densidade da água.

A caracterização microestrutural foi feita por meio de microscopia eletrônica de varredura (Philips XL30 FEG).

\section{RESULTADOS E DISCUSSÃO}

A fim de estabelecer a curva de queima, foram realizadas análise térmica diferencial (ATD) e análise termogravimétrica (TG) para a massa plástica de alumina utilizada neste trabalho. Com a curva da análise térmica diferencial pode-se observar que o intervalo de temperatura na qual ocorre a eliminação dos compostos orgânicos, utilizados para a preparação da massa plástica de alumina, termina por volta dos $650{ }^{\circ} \mathrm{C}$. Os picos endotérmicos a aproximadamente $134{ }^{\circ} \mathrm{C}, 245{ }^{\circ} \mathrm{C}$ e $400{ }^{\circ} \mathrm{C}$ referem-se à decomposição e volatilização do lubrificante, veículo (solvente) e plastificante, respectivamente. Dessa forma, para garantir a saída de todo os voláteis um patamar de 20 min foi estabelecido a $700{ }^{\circ} \mathrm{C}$. O gráfico com as curvas de ATD e TG é apresentado na Fig. 2.

Geralmente, o substrato cerâmico para suporte de membranas requer uma porosidade total em torno de $45 \%$

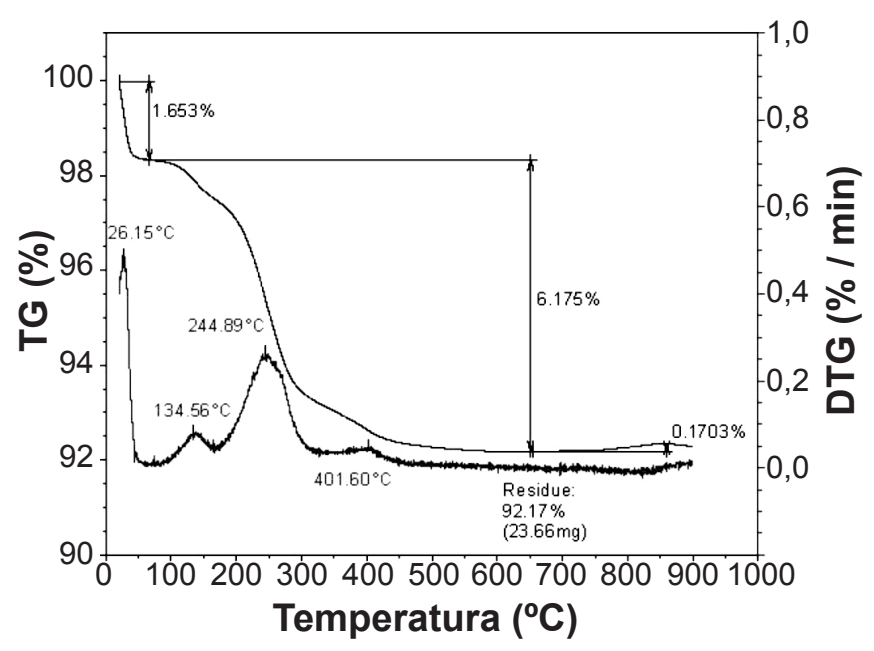

Figura 2: Curvas de análise térmica diferencial e análise termogravimétrica da massa plástica de alumina para a conformação de substratos cerâmicos.

[Figure 2: DTA and TG thermal analysis curves of alumina plastic paste to conform ceramic substrates.]

[14]. Neste trabalho foram obtidos substratos cerâmicos porosos de alumina com porosidade aparente de $21 \%$ e $52 \%$ para seguintes concentrações de grafite, $9 \%$ e $18 \%$ em massa, respectivamente. Assim como o esperado, é possível verificar pela Fig. 3 e Fig. 4, respectivamente, que tanto a densidade quanto a porosidade aparente foram alteradas pelos diferentes teores de grafite adicionados a massa plástica para conformação de substratos porosos.

\section{Densidade aparente X Composição}

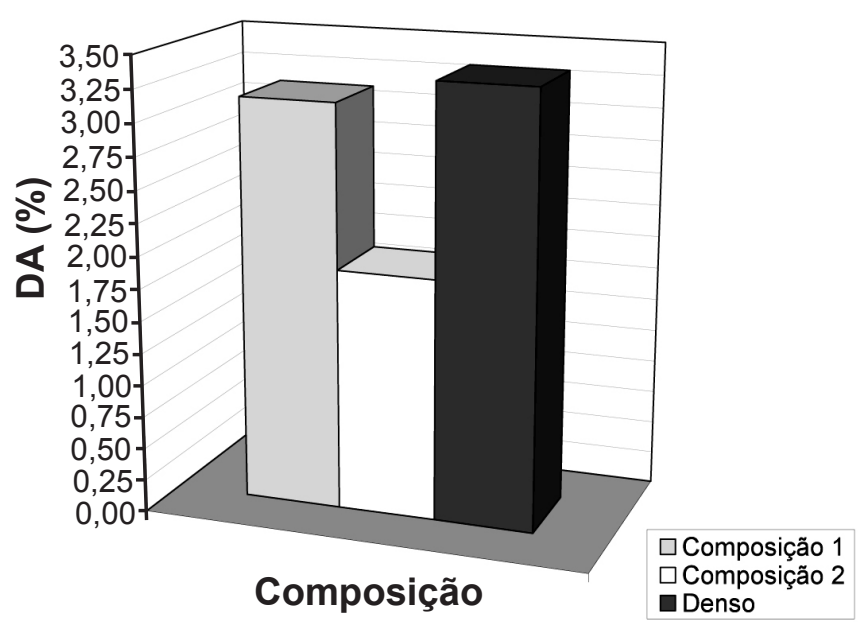

Figura 3: Gráfico comparativo da densidade aparente entre os substratos porosos de alumina com $9 \%$ e $18 \%$ de agente porogênico e o substrato denso de alumina, conformados por rolos a frio e sinterizados a $1600{ }^{\circ} \mathrm{C}$.

[Figure 3: Comparative graphic between apparent densities of alumina porous substrates with $9 \%$ and $18 \%$ of porous forming agent and alumina dense substrates conformed by cold roll pressing and fired at $1600^{\circ} \mathrm{C}$.] 


\section{Porosidade aparente X Composição}

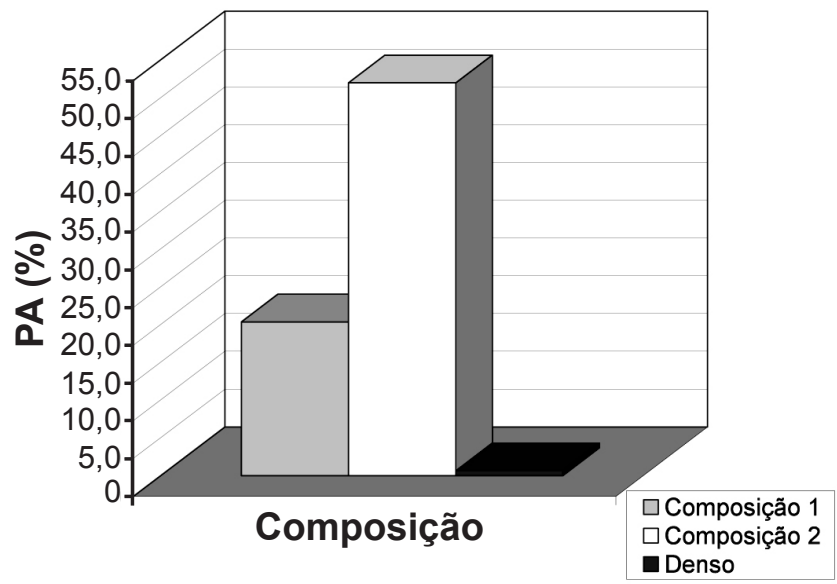

Figura 4: Gráfico comparativo da porosidade aparente entre os substratos porosos de alumina com $9 \%$ e $18 \%$ de agente porogênico e o substrato denso de alumina, conformados por rolos a frio e sinterizados a $1600{ }^{\circ} \mathrm{C}$.

[Figure 4: Comparative graphic between apparent porosity of alumina porous substrates with $9 \%$ and $18 \%$ of porous forming agent and alumina dense substrates conformed by cold roll pressing and fired at $1600{ }^{\circ} \mathrm{C}$.]

Para um maior teor de grafite em pó, maior a porosidade e menor a densidade da amostra, uma vez que a grafite, utilizada como agente porogênico, é eliminada durante a sinterização deixando espaços vazios na matriz cerâmica.

Além das diferenças nas propriedades físicas dos substratos de alumina porosos com diferentes teores de grafite, foi notada empiricamente uma maior dificuldade na homogeneização da grafite com maior concentração (18\%), devido a sua baixa molhabilidade e ao efeito lubrificante das partículas. Já com relação à trabalhabilidade não

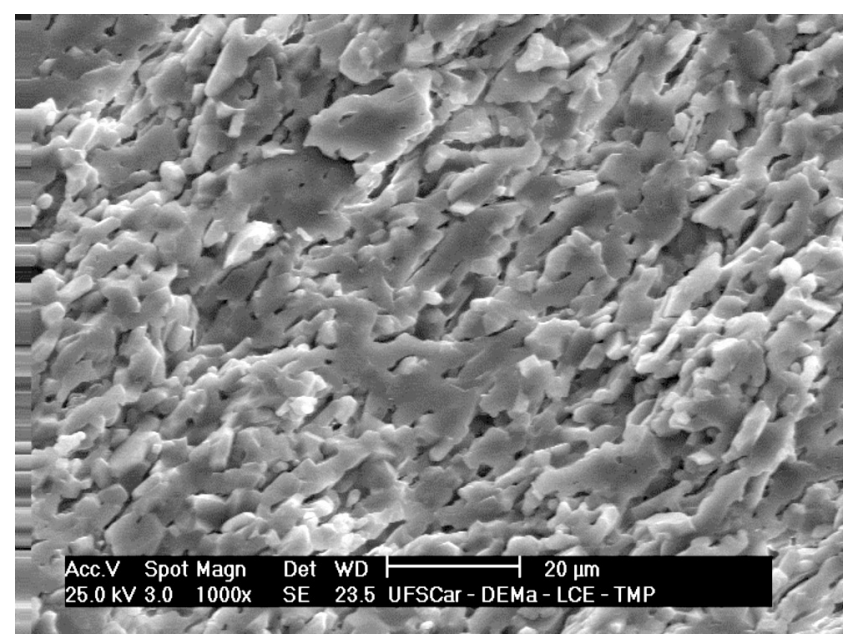

Figura 5: Micrografia por microscopia eletrônica de varredura da superfície do substrato poroso de alumina com $9 \%$ do agente porogênico grafite conformado por rolos a frio e sinterizado a $1600{ }^{\circ} \mathrm{C}$.

[Figure 5: Scanning electron microscopy micrograph of alumina porous substrate surface with $9 \%$ of graphite porous forming agent conformed by cold roll pressing and fired at $1600{ }^{\circ} \mathrm{C}$.] houve diferença significativa, sendo que ambas as massas apresentaram plasticidade suficiente para a conformação e comportaram de maneira semelhante durante o processamento e a queima.

Conforme mostram as Figs. 5 e 6, respectivamente, a microestrutura da superfície dos substratos porosos de alumina com adição de grafite de $9 \%$ difere na quantidade de poros e na coesão das partículas de alumina com relação à microestrutura do substrato com $18 \%$ de grafite. Esta diferença se deve à variação no teor de grafite adicionado a massa de alumina, sendo que quanto maior esse valor, mais vazios será gerado após a queima, tornando a microestrutura

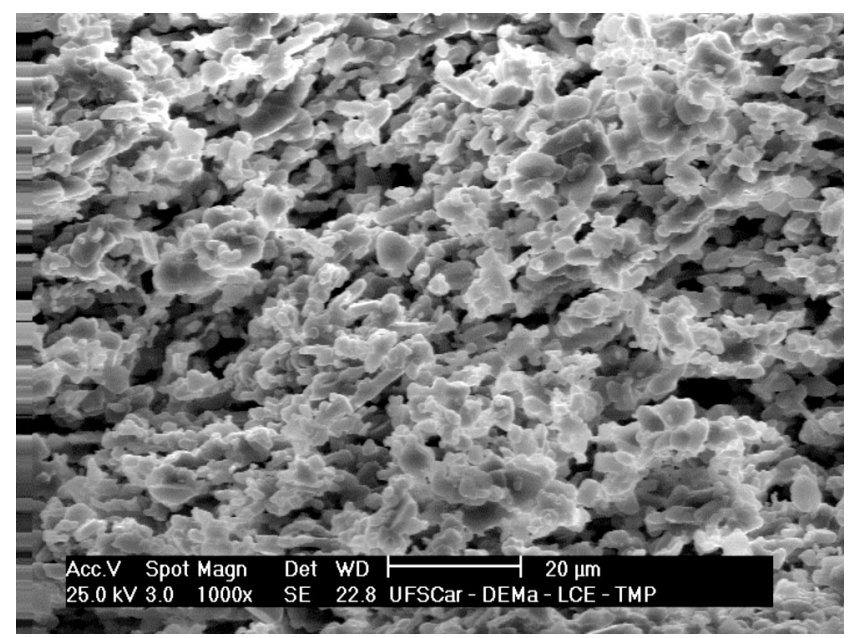

Figura 6: Micrografia por microscopia eletrônica de varredura da superfície do substrato poroso de alumina com $18 \%$ do agente porogênico grafite conformado por rolos a frio e sinterizado a $1600{ }^{\circ} \mathrm{C}$.

[Figure 6: Scanning electron microscopy micrograph of alumina porous substrate surface with $18 \%$ of graphite porous forming agent conformed by cold roll pressing and fired at $\left.1600{ }^{\circ} \mathrm{C}.\right]$

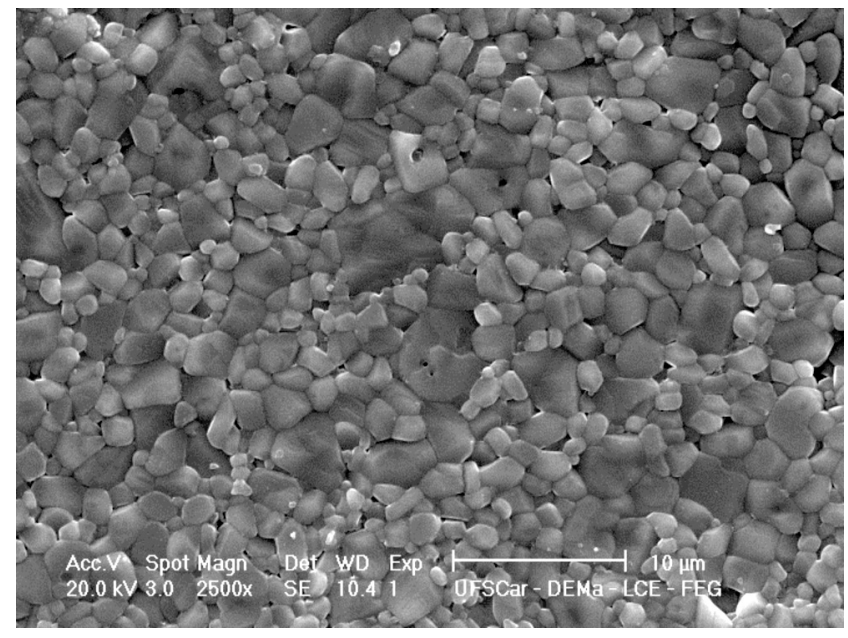

Figura 7: Micrografia por microscopia eletrônica de varredura da superfície de substrato denso de alumina conformado por rolos a frio e sinterizado a $1600{ }^{\circ} \mathrm{C}$.

[Figure 7: Scanning electron microscopy micrograph of alumina dense substrate surface conformed by cold roll pressing and fired at $\left.1600{ }^{\circ} \mathrm{C}.\right]$ 
mais porosa e com menor coesão entre as partículas. Quando comparadas as microestruturas dos substratos porosos, Fig. 5 e Fig. 6, à microestrutura do substrato denso de alumina, Fig. 7, assim como esperado, é possível notar a diferença com relação à porosidade devido à adição do agente porogênico grafite na massa plástica de alumina. De acordo com literatura de outros estudos realizados para a obtenção de substratos cerâmicos porosos, este método alternativo de conformação plástica por rolos a frio mostra-se viável e competitivo quando comparado aos métodos tradicionais atualmente utilizados. É mostrado que também foram produzidos substratos cerâmicos porosos, porém foi utilizado o carbono como agente porogênico e o método de colagem de fita, sendo que a espessura mínima obtida foi de $1400 \mu \mathrm{m}$ [15].

\section{CONCLUSÃO}

Com o método de processamento viscoplástico foram conformados substratos porosos de alumina, com espessuras variáveis entre 400 a $700 \mu \mathrm{m}$, confirmando a viabilidade do processo para a obtenção substratos porosos cerâmicos de fina espessura. A variação nas composições com relação ao teor do agente porogênico, grafite em pó foi determinante nas propriedades físicas finais dos substratos porosos, sendo que para maiores concentrações (18\%), apesar da maior a dificuldade para a homogeneização da massa plástica de alumina, obteve-se maior porosidade (menor densidade aparente) sem a ocorrência de distorções, trincas ou rupturas. Além da alteração nas densidades e porosidades dos substratos porosos, a variação no teor da grafite adicionado a massa de alumina também modificou a microestrutura final das amostras. O teor de $18 \%$ de grafite em massa foi o que promoveu a porosidade mais próxima à necessária para aplicações em membranas cerâmicas.

\section{AGRADECIMENTOS}

Ao Programa de Pós - Graduação em Ciência e
Engenharia de Materiais (PPG-CEM) da UFSCar e ao CNPq pelo suporte e apoio financeiro.

\section{REFERÊNCIAS}

[1] O. Lyckfeldt, J. M. F. Ferreira, J. Eur. Ceram. Soc. 18 (1998) 131.

[2] R. Ghidossi, E. Carretier, D. Veyret, D. Dhaler, P. Moulin, J. Membrane Sci. 360 (2010) 483.

[3] H. M. Alves, G. Tari, A. T. Fonseca, J. M. F. Ferreira, Mater. Res. Bull. 33, 10 (1998) 1439.

[4] Y. H. Wang, Y. Zhang, X. Liu, G. Y. Meng, Powder Technol. 168 (2006) 125.

[5] C. Galassi, J. Eur. Ceram. Soc. 26 (2006) 2951.

[6] R. W. Rice, Ceramic fabrication technology, Marcel Dekker, Inc., EUA (2003).

[7] S. Li, N. Li, Y. Li, Ceram. Int. 34 (2008) 1241.

[8] S. Li, N. Li, Ceram. Int. 33 (2007) 551.

[9] S. Bhattacharjee, L. Besra, B. P. Singh, J. Eur. Ceram. Soc. 27 (2007) 47.

[10] A. F. R. Pardo, Processamento viscoplástico e conformação cerâmica por rolos a frio a partir de suspensões concentradas de alumina, Tese Dr., Departamento de Engenharia de Materiais, UFSCar, S. Carlos, SP (2005).

[11] F. A. Mesquita, M. R. Morelli, J. Mater. Proc. Technol. 143-144 (2003) 232.

[12] Z. Zivcová, M. Cerný, W. Pabst, E. Gregorová, J. Eur. Ceram. Soc. 29 (2009) 2765.

[13] L. Koshimizu, Conformação viscoplástica por rolos a frio e caracterização de substratos de alumina, Diss. Mestrado, Departamento de Engenharia de Materiais, UFSCar, S. Carlos, SP (2008).

[14] F. Bouzerara, A. Harabis, S. Achour, A. Larbot, J. Eur. Ceram. Soc. 26 (2006) 1663.

[15] H. L. Lein, T. Tezuka, T. Grande, M. A. Einarsrud, Solid State Ionics 179 (2008) 1146.

(Rec. 18/11/2011, Ac. 25/01/2012) 